\title{
The Role of Syk in Inflammatory Response of Human Abdominal Aortic Aneurysm Tissue
}

\author{
Ryo Kanamoto, MD, ${ }^{1}$ Hiroki Aoki, MD, PhD, ${ }^{2}$ Aya Furusho, MD, PhD, ${ }^{3}$ \\ Hiroyuki Otsuka, MD, PhD, ${ }^{1}$ Yusuke Shintani, MD, PhD, ${ }^{1}$ Satoru Tobinaga, MD, PhD, ${ }^{1}$ \\ Shinichi Hiromatsu, MD, PhD, ${ }^{1}$ Yoshihiro Fukumoto, MD, PhD, ${ }^{3}$ and \\ Hiroyuki Tanaka, MD, PhD'
}

Objective: Inflammatory response is central to pathogenesis of abdominal aortic aneurysm (AAA). Recently, we reported that Syk, a signaling molecule in inflammatory cells, promotes AAA development in a mouse model. In this study, we aimed to investigate the role of Syk in human AAA pathogenesis.

Materials and Methods: We obtained human AAA wall samples during open surgical aortic repair at Kurume University Hospital. Immunohistochemical analyses of AAA samples were performed for Syk activation and cell type markers. Ex vivo culture of human AAA tissue was utilized to evaluate the effect of P505-15, a Syk inhibitor, on secretions of interleukin-6 (IL-6) and matrix metalloproteinases (MMPs).

Results: Immunohistochemical analysis showed infiltration of B cells, T cells, and macrophages in AAA samples. Syk activation was localized mainly in B cells and part of macrophages. AAA tissue in culture secreted IL-6, MMP-9, and MMP-2 without any stimulation. The unstimulated secretions of IL-6, MMP-9, and MMP-2 were insensitive to P505-15. Secretions of IL-6 and MMP-9 were enhanced by exogenous normal human immunoglobulin G (lgG), which

${ }^{1}$ Division of Cardiovascular Surgery, Department of Surgery, Kurume University School of Medicine, Kurume, Fukuoka, Japan

${ }^{2}$ Cardiovascular Research Institute, Kurume University, Kurume, Fukuoka, Japan

${ }^{3}$ Division of Cardiovascular Medicine, Department of Internal Medicine, Kurume University School of Medicine, Kurume, Fukuoka, Japan

Received: January 15, 2020; Accepted: March 10, 2020 Corresponding author: Hiroki Aoki, MD, PhD. Cardiovascular Research Institute, Kurume University, 67 Asahimachi, Kurume, Fukuoka 830-0011, Japan

Tel: +81-942-31-7880, Fax: +81-942-31-7707

E-mail: haoki@med.kurume-u.ac.jp

(cc) BY-NC-SA (02020 The Editorial Committee of Annals of Vascular Diseases. This article is distributed under the terms of the Creative Commons Attribution License, which permits use, distribution, and reproduction in any medium, provided the credit of the original work, a link to the license, and indication of any change are properly given, and the original work is not used for commercial purposes. Remixed or transformed contributions must be distributed under the same license as the original. was suppressed by P505-15, whereas secretion of MMP-2 was insensitive to IgG or P505-15.

Conclusion: These results demonstrate an important role of Syk for IgG-dependent inflammatory response in human AAA.

Keywords: abdominal aortic aneurysm, inflammation, Syk

\section{Introduction}

Abdominal aortic aneurysm (AAA) is common among elderly people, which is caused by the local weakening of aortic walls. ${ }^{1)}$ Although AAA usually presents no symptoms, it leads to the progressive dilation and abrupt rupture of the aorta with high mortality. To prevent the lethal event of aortic rupture, therapeutic options are either replacement of the weakened part of the aorta with an artificial graft by open surgery or insertion of a self-expandable stent graft spanning the aneurysmal lesion through a catheter, termed as endovascular aneurysm repair. ${ }^{2)}$ Other than these surgical techniques, no medical therapy has been established to prevent the progression and rupture of AAA.

Accumulating evidence indicate that chronic inflammation is central to tissue destruction in AAA. ${ }^{3,4)}$ Involvement of inflammation is underscored by the infiltration of inflammatory cells including $\mathrm{B}$ cells, $\mathrm{T}$ cells, and macrophages in AAA tissue before the destruction of extracellular matrix and expansion of the aorta. ${ }^{1)}$ Among the inflammatory cells, we and others demonstrated that $B$ cells and their effector molecule IgG promote AAA in mouse models of AAA. ${ }^{5-7)}$ We also demonstrated that human AAA tissue secretes a number of inflammatory cytokines including interleukin-6 (IL-6), ${ }^{8)}$ and exogenously added IgG promotes the secretions of IL- 6 and matrix metalloproteinase-9 (MMP-9) from human AAA tissue in culture. ${ }^{7)}$

Although B cells can theoretically be a therapeutic target for AAA, depletion or functional suppression of $B$ cells in patients is impractical, because of the fact that 
Table 1 Clinical characteristics of patients in abdominal aortic aneurysm (AAA) tissue culture

\begin{tabular}{|c|c|c|c|c|c|c|c|}
\hline Case \# & Age & $\begin{array}{c}\text { Sex } \\
\text { (male/female) }\end{array}$ & $\begin{array}{l}\text { Maxi. diameter } \\
\qquad(\mathrm{mm})\end{array}$ & Location of AAA & Comorbidity & Smoking & Medication \\
\hline TC \#1 & 76 & Male & 92 & $\begin{array}{l}\text { Infrarenal- } \\
\text { Common iliac artery }\end{array}$ & None & Current & None \\
\hline TC \#2 & 86 & Female & 67 & Infrarenal-Terminal aorta & HT & None & CCB \\
\hline TC \#3 & 82 & Female & 55 & $\begin{array}{l}\text { Infrarenal- } \\
\text { Common iliac artery }\end{array}$ & DL, HT, IHD & None & ARB, APT, CCB, Statin \\
\hline TC \#4 & 58 & Male & 58 & Infrarenal-Terminal aorta & CVD, DL, HT, IHD & History & ARB, APT, Statin \\
\hline TC \#5 & 85 & Male & $48^{*}$ & Infrarenal-Terminal aorta & CKD, DL, HT, IHD & History & ARB, BAB, APT, Statin \\
\hline TC \#6 & 73 & Male & $48^{* *}$ & $\begin{array}{l}\text { Infrarenal- } \\
\text { Common iliac artery }\end{array}$ & CKD, DL, DM, HT, IHD & None & ARB, CCB, Statin \\
\hline TC \#7 & 73 & Male & 57 & $\begin{array}{l}\text { Infrarenal- } \\
\text { Common iliac artery }\end{array}$ & CVD, DL, Graves, IHD & Current & ACEi, APT, BAB, Statin \\
\hline HP \#1 & 75 & Male & $48^{* *}$ & $\begin{array}{l}\text { Infrarenal- } \\
\text { Common iliac artery }\end{array}$ & DL, IHD & History & APT, Statin \\
\hline HP \#2 & 82 & Male & 50 & Infrarenal-Terminal aorta & HT, DL, IHD & Current & ACEi, APT, BAB, Statin \\
\hline$\underline{\mathrm{HP} \# 3}$ & 62 & Male & 54 & Infrarenal-Terminal aorta & $\mathrm{DL}$ & History & Statin \\
\hline HP \#4 & 68 & Female & 54 & Infrarenal-Terminal aorta & $\mathrm{DL}, \mathrm{DM}, \mathrm{HCM}, \mathrm{HT}$ & None & ARB, Statin, Isosorbide \\
\hline
\end{tabular}

Clinical characteristics of the patients of whom AAA tissue was obtained for tissue culture (TC) or histopathology (HP). HP \#3 sample was used for the representative data in Figs. 1 and 2.

CKD: chronic kidney disease; CVD: cerebrovascular disease; DL: dyslipidemia; DM: diabetes mellitus; HCM: hypertrophic cardiomyopathy; HT: hypertension; IHD: ischemic heart disease; ACEi: angiotensin-converting enzyme inhibitor; ARB: angiotensin II type 1 receptor blocker; APT: antiplatelet drugs; BAB: $\beta$-adrenergic blocker; CCB: calcium channel blocker

*Surgical repair was carried out for TC \#5 due to the concern of the advanced frailty and surgical intolerability of the patient if the surgery is to be carried out after the diameter reaches $5 \mathrm{~cm}$. ${ }^{* *}$ Surgical repair was carried out for TC \#6 and HP \#1 to repair the iliac artery aneurysms with diameters exceeding $3 \mathrm{~cm}$ together with the AAAs.

AAA is a chronic disease that persists for several years without symptoms and the concern for immunosuppression. In addition, it is difficult to fine tune the dose and the effect according to the disease activity or the adverse side effects using the current antibody-based medicine to deplete $\mathrm{B}$ cells, such as anti-CD20 monoclonal antibody rituximab. ${ }^{9)}$

Considering the flexibility in drug use, the non-receptor tyrosine kinase Syk is an attractive candidate drug target, because Syk plays a central role both in B cell function and in immunoglobulin effector function, ${ }^{10-13)}$ and small-molecule Syk inhibitors are available. Indeed, we previously reported that a Syk inhibitor can suppress AAA development in mice. ${ }^{7)}$ However, no mouse model can recapitulate all aspects of human AAA. ${ }^{14)}$ Therefore, in this study, we intended to test the effect of a Syk inhibitor in human AAA tissue in ex vivo culture that maintains inflammatory and tissue destructive activities for several days. ${ }^{15}$ )

\section{Materials and Methods}

\section{Human AAA wall tissue}

All protocols that involved human specimens were approved by the Institutional Review Board at Kurume University Hospital (approval number 16139), and all samples were obtained with written informed consent from the patients. Human AAA tissue was obtained from patients during open surgery performed to repair AAA (Table 1). Tissue was acquired from the anterior wall of the aneurysm.

\section{Histological analysis}

For histological analyses, AAA tissues were fixed in paraformaldehyde, paraffin embedded, and sliced into 5 - $\mu$ m-thick tissue sections. We stained paraffin-embedded sections of human AAA tissue with Elastica van Gieson (EVG) or hematoxylin and eosin (H\&E). We performed immunohistochemical staining of human AAA tissue with antibodies specific for activated (phosphorylated) Syk (pSyk, phospho-Y323, Abcam, Cambridge, UK; \#ab63515, $20 \mu \mathrm{g} / \mathrm{mL}$ ), B cells (CD20, Leica Biosystems, Wetzlar, Germany; \#NCL-L-CD20-L26, $80 \mu \mathrm{g} / \mathrm{mL}$ ), T cells (CD3, Leica Biosystems; \#NCL-L-CD3-565, $105 \mu \mathrm{g} / \mathrm{mL}$ ), smooth muscle cells (smooth muscle $\alpha$-actin, Dako, Jena, Germany; \#M0851, $180 \mu \mathrm{g} / \mathrm{mL}$ ), and macrophages (CD68, Dako; \#M0876, $155 \mu \mathrm{g} / \mathrm{mL}$ ). To examine the tissue localization of activated Syk and other inflammatory cells, immunofluorescence staining of the human AAA tissue was performed. An antibody for pSyk (phospho-Y323, Abcam; \#ab63515, $40 \mu \mathrm{g} / \mathrm{mL}$ ) was used for detecting activated Syk visualized by indirect immunofluorescence staining with the Tyramide Signal Amplification labeling 
Table 2 Well numbers obtained from each patient for tissue culture

\begin{tabular}{|c|c|c|c|c|c|}
\hline \multirow{2}{*}{ Case \# } & \multicolumn{4}{|c|}{ Experimental interventions } & \multirow{2}{*}{ Assays } \\
\hline & Control & P505-15 & $\lg G$ & $\lg G+P 505-15$ & \\
\hline TC \#1 & 4 & 4 & $\mathrm{~N}$ & $\mathrm{~N}$ & MMP-9/MMP-2, IL-6 \\
\hline TC \#2 & 4 & 4 & $\mathrm{~N}$ & $\mathrm{~N}$ & MMP-9/MMP-2 \\
\hline TC \#3 & 4 & $\mathrm{~N}$ & 4 & 4 & MMP-9/MMP-2, IL-6 \\
\hline TC \#4 & 4 & 4 & 4 & 4 & MMP-9/MMP-2, IL-6 \\
\hline TC \#5 & 4 & 4 & 4 & 4 & MMP-9/MMP-2, IL-6 \\
\hline TC \#6 & 5 & 5 & 5 & 5 & MMP-9/MMP-2 \\
\hline TC \#7 & 2 & 2 & 2 & 2 & MMP-9/MMP-2 \\
\hline
\end{tabular}

Abdominal aortic aneurysm samples were used to obtain the indicated number of wells of 6-well tissue culture plate for the assays of matrix metalloproteinase (MMP)- 9 and MMP-2 by gelatin zymogram and interleukin- 6 by enzyme-linked immunosorbent assay. N: not used for the indicated experimental condition

kit with Alexa-Fluor 488 tyramide (\#T-20922, Thermo Fisher Scientific, Waltham, MA, USA). Antibodies for CD20 (Leica Biosystems; \#NCL-L-CD20-L26, $95 \mu \mathrm{g} / \mathrm{mL}$ ), CD3 (Leica Biosystems; \#NCL-L-CD3-565, $80 \mu \mathrm{g} / \mathrm{mL}$ ), and CD68 (CD68, Dako; \#M0876, 370 $\mu \mathrm{g} / \mathrm{mL}$ ) were used for detecting $\mathrm{B}$ cells, $\mathrm{T}$ cells, and macrophages, respectively, by indirect immunofluorescence staining with Cy3conjugated goat anti-mouse IgG antibody (\#715-165-151, Jackson ImmunoResearch Laboratories, West Grove, PA, USA). TO-PRO-3 (\#T3605, Thermo Fisher Scientific) was used for nuclear staining.

\section{AAA tissue culture}

The ex vivo culture experiment of human AAA tissue was performed as previously described. ${ }^{15)}$ Briefly, we obtained fresh AAA samples including the site of maximal diameter from patients during open surgery performed to repair AAA that were cleaned of thrombi and loose connective tissue. The AAA tissue was cut horizontally into three parts. Each part of the aortic wall was minced into pieces of approximately $2 \times 2 \mathrm{~mm}$ with maintaining full thickness of the aortic wall. Minced tissues were transferred to 12-well plates with serum-free Dulbecco's Modified Eagle Medium (DMEM) (\#11885-084, Invitrogen, Carlsbad, CA, USA). Into each well, we placed an equal number of pieces from all three parts of the AAA sample to minimize the effect of AAA tissue heterogeneity. At $48 \mathrm{~h}$ after starting the culture, culture media were exchanged with fresh DMEM, and samples were incubated for $48 \mathrm{~h}$ (the first $48 \mathrm{~h}$, Pre values). We obtained conditioned media from the first $48 \mathrm{~h}$ of culture for measuring basal secretions of IL-6 and MMP-9 and MMP-2 (Table 2). Then, culture media were exchanged with fresh DMEM, with or without $0.5 \mathrm{mg} / \mathrm{mL}$ human polyclonal IgG (Jackson ImmunoResearch, West Grove, PA; \#767-71594) and $10 \mu \mathrm{mol} / \mathrm{L}$ P505-15 (Selleckchem.com, Houston, TX, USA), a specific Syk inhibitor, and cultures were continued for another $48 \mathrm{~h}$ (the second $48 \mathrm{~h}$, Post values) to obtain conditioned media after stimulation. The conditioned media after stimulation was used for measuring stimulated secretions of IL-6 and MMPs. Secreted IL-6 and MMPs were measured with an IL-6 enzyme-linked immunosorbent assay (ELISA) kit (R\&D Systems, Minneapolis, MN, USA; \#S6050). Secreted MMP-2 and MMP-9 were measured by gelatin zymography (Thermo Fisher Scientific, Waltham, MA, USA; \#EC61755BOX). To normalize the variability in the tissue, we calculated the ratios of the stimulated secretion during the second $48 \mathrm{~h}$ (Post values) and the basal secretion during the first $48 \mathrm{~h}$ (Pre values) to evaluate the effect of experimental interventions.

\section{Statistical analysis}

All data are expressed as individual data points with the median \pm interquartile range relative to the control sample without any experimental intervention. All analyses were performed using JMP 13 (SAS Institute, Cary, NC, USA). To compare three or more groups, the Kruskal-Wallis test followed by Dunn's post hoc test was used. Statistical significance was accepted by a P value of less than 0.05 .

\section{Results}

\section{Activation of Syk in human AAA tissue}

We first examined the histological characteristics and activation status of Syk in human AAA tissue obtained from patients who underwent open surgery to repair AAA (Fig. 1, Table 1). The AAA tissues showed loss of elastic fibers in EVG staining and infiltration of inflammatory cells as observed in H\&E staining (Fig. 1B). Immunohistochemical staining of smooth muscle $\alpha$-actin, a marker of smooth muscle cells (SMCs), showed disruption and disarray of SMC layers. Immunohistochemical staining of inflammatory cell markers, including CD20 (B cells), CD3 ( $\mathrm{T}$ cells), and CD68 (macrophages), indicated that these cell types make cell clusters adjacent to SMC layer in the aortic walls (Fig. 1C). Immunostaining for phosphorylated and hence activated Syk (pSyk) showed that cells positive for $\mathrm{pSyk}$ are in the cluster of inflammatory cells. 

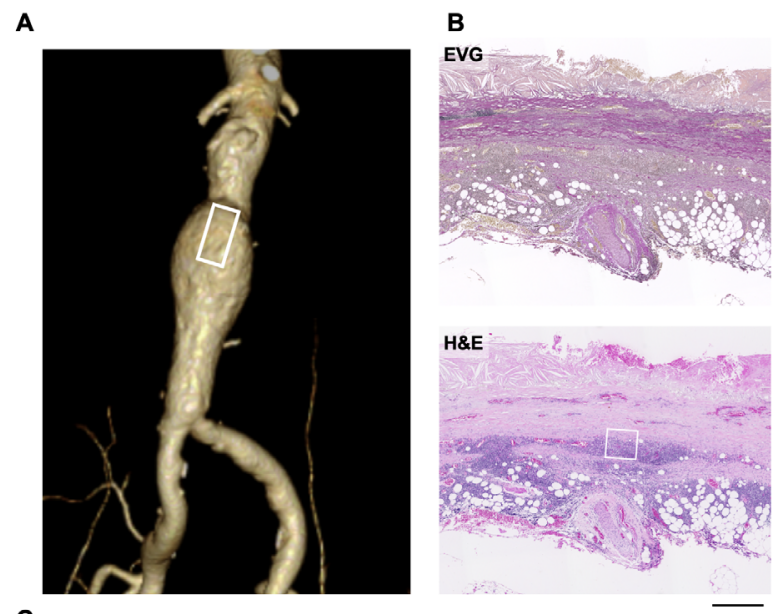

C

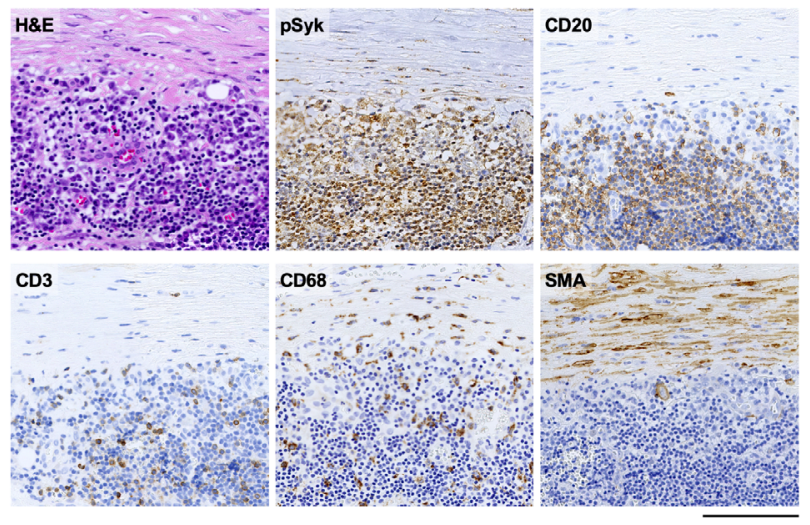

Fig. 1 Histological analysis of human abdominal aortic aneurysm (AAA) wall tissue.

(A) A three-dimensional computed tomography image is shown for the representative AAA case. A white rectangle indicates the location of tissue curation for the analysis. (B) Low-magnification images of human AAA wall tissue with Elastica van Gieson and hematoxylin and eosin (H\&E) staining. Bar, $0.5 \mathrm{~mm}$. A white rectangle indicates the area corresponding to the high-magnification images. (C) High-magnification images of H\&E staining and immunohistochemical staining for activated (phosphorylated) Syk (pSyk) and cell type markers, CD20 (B cells), CD3 (T cells), CD68 (macrophages), and smooth muscle a-actin (SMA; smooth muscle cells). Bar, $0.1 \mathrm{~mm}$. All images are shown with the luminal side up and adventitial side down.

To further characterize pSyk-positive cell types in human AAA tissue, we performed double immunofluorescence staining for pSyk and cell type markers (CD20, CD3, and CD68) (Fig. 2). In the observed area, CD20-positive $\mathrm{B}$ cells were most abundant, making tight cell clusters. CD3-positive T cells were scattered in the cell clusters, and CD68-positive macrophages were more sparse within the cell clusters. pSyk staining overlapped mainly with CD20positive $\mathrm{B}$ cells and part of CD68-positive macrophages, whereas the overlap with CD3 was less obvious. Other samples from three other patients showed similar results. These results suggested that Syk was activated mainly in B

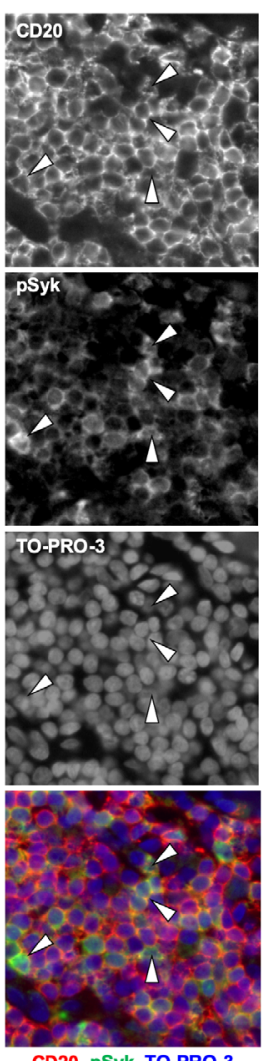

CD20 pSyk TO-PRO-3
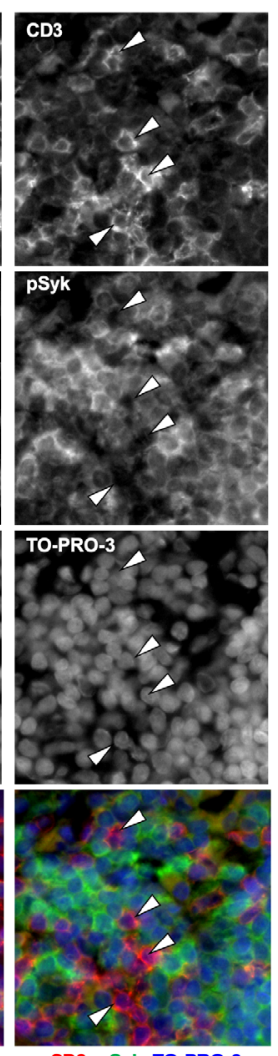

CD3 pSyk TO-PRO-3

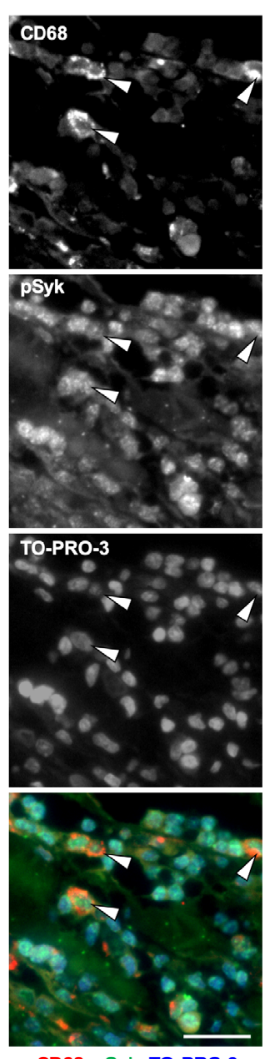

CD68 pSyk TO-PRO-3
Fig. 2 Localization of pSyk and cell type markers. Immunofluorescence images for cell type markers for B cells (CD20), T cells (CD3), and macrophages (CD68), activated Syk (pSyk), and nuclei by TO-PRO-3. The overlap images of pSyk (green) and cell type markers (red) and nuclei (blue). Arrowheads indicate the cells with the corresponding cell type markers. Bar, $20 \mu \mathrm{m}$.

cells and part of macrophages in AAA tissue.

\section{Involvement of Syk in AAA pathogenesis}

To investigate the role of Syk in AAA pathogenesis, we made ex vivo tissue culture of human AAA tissue (Table 1) and measured the secreted MMP-9 and MMP-2 to assess the tissue destructive activity (Table 2). Human AAA tissue was obtained from seven randomly chosen patients during open surgery performed for AAA repair. We excluded the patients with family history of AAA to exclude the familial forms of AAA. We also excluded suprarenal AAA or ruptured AAA. Human AAA tissue secreted both MMP-2 and MMP-9 in the culture media (Fig. 3A). Exogenously added human IgG stimulated the secretion of MMP-9, but not that of MMP-2. P505-15 showed no effect on unstimulated secretion of MMP-9 but suppressed IgG-stimulated secretion of MMP-9. MMP-2 secretion was insensitive to either IgG or P-505-15. To evaluate the inflammatory activity of AAA tissue, we measured the secretion of IL-6 (Fig. 3B). P505-15 showed no effect on the unstimulated secretion of IL-6 from AAA tissue. 
A
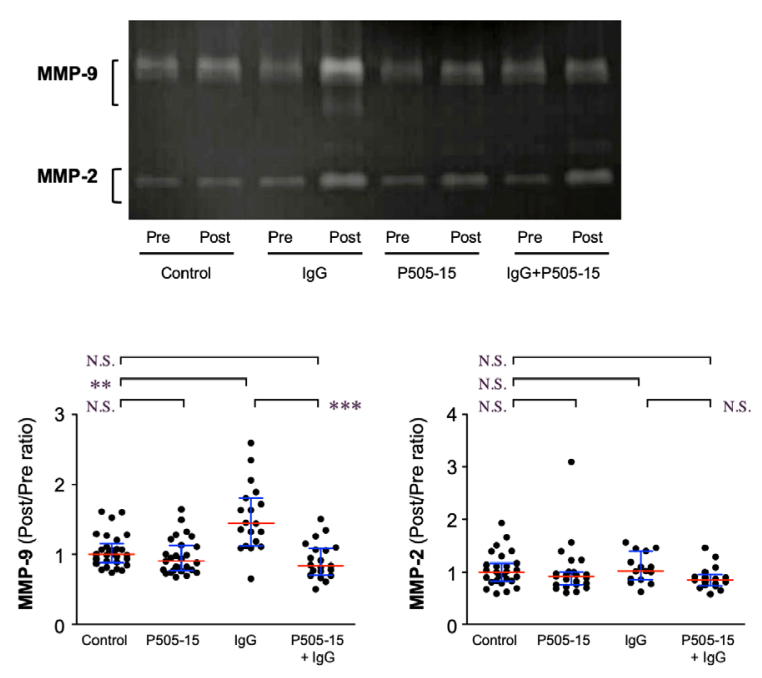

B

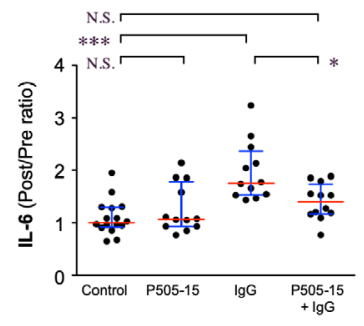

Fig. 3 Secretions of matrix metalloproteinases (MMPs) and interleukin-6 (IL-6) in ex vivo culture of human abdominal aortic aneurysm (AAA) tissue.

(A) Secretions of MMP-2 and MMP-9 from human AAA tissue culture in the presence or absence of P505-15, a specific Syk inhibitor, and normal human immunoglobulin $\mathrm{G}$ (IgG). MMPs were measured by gelatin zymogram for the basal secretion (Pre) and the stimulated secretion (Post). A representative image of the zymogram is shown. The graph shows the ratios of the stimulated secretion and the basal secretion for MMP-9 and MMP-2. (B) Secretion of IL-6 was measured by enzyme-linked immunosorbent assay in the same way as MMPs. Red and blue bars indicate medians and interquartile ranges. N.S.: not significant; ${ }^{*} \mathrm{P}<0.05,{ }^{* *} \mathrm{P}<0.01,{ }^{* * *} \mathrm{P}<0.001$.

Exogenously added IgG stimulated the secretion of IL-6 into the culture media, ${ }^{7)}$ which was effectively suppressed by P505-15. These findings indicated that Syk was involved in IgG-stimulated secretions of IL-6 and MMP-9 in human AAA tissue.

\section{Discussion}

In this study, we showed in human AAA tissue that infiltrating inflammatory cells, including B cells, $\mathrm{T}$ cells, and macrophages, are making cell clusters, suggesting the cellcell interaction of these cell types. Among the cell types of inflammatory cells and smooth muscle cells, activated Syk was located mainly in B cells, underscoring its importance in $\mathrm{B}$ cell activity. ${ }^{10,12,13)}$ Of note, we demonstrated that P505-15, a specific Syk inhibitor, effectively suppressed the secretions of IL- 6 and MMP-9 that were induced by exogenous IgG, supporting the notion that Syk is a potential therapeutic target for AAA.

\section{Pathogenetic role of $B$ cells and IgG in human AAA}

While it is well established that AAA tissue is enriched for $\mathrm{B}$ cells and immunoglobulins, ${ }^{16-18)}$ their roles in AAA pathogenesis is still under investigation. Recently, a series of reports have uncovered the mechanism by which $\mathrm{B}$ cells and immunoglobulins promote AAA progression in mice. ${ }^{5,6,19)}$ In these reports, it has been shown that naturally occurring IgG in normal mouse serum contains anti-fibrinogen antibodies that can bind to the deposited fibrinogen in the injured aortic tissue, which initiates the local inflammatory response. Consistently, we found that exogenously added normal human IgG promoted secretions of IL-6 and MMP-9 from human AAA tissue in ex vivo culture in previous study ${ }^{7}$ and in the current study. Different lots of normal human IgG in our studies stimulated IL-6 and MMP-9 secretions to similar extent. Similarly, different lots of normal mouse IgG promoted AAA in B cell- and immunoglobulin-deficient $\mu \mathrm{MT}$ mice, ${ }^{7)}$ suggesting that naturally occurring antibodies are responsible for the inflammatory effect. Whether such stimulating IgG is against fibrinogen, similar to the mouse model in the previous study, ${ }^{5)}$ or have other antigens remains to be elucidated.

\section{Action point of the Syk}

With regard to the function of $\mathrm{B}$ cells and immunoglobulins, the main function of Syk is the B cell receptor (BCR)mediated regulation of $\mathrm{B}$ cells and the signal transduction under the Fc receptors of immunoglobulins. In B cells, engagement of BCR with corresponding antigen activates signal pathway involving Syk ${ }^{11)}$ and regulates development and activation of B cells. ${ }^{20)}$ In inflammatory cell, $\mathrm{Fc} \gamma$ receptors mediate IgG-dependent response, where immunoreceptor tyrosine-based activation motif-containing Fc $\gamma$ receptors mediate activation signal through Syk, whereas immunoreceptor tyrosine-based inhibitory motifcontaining $\mathrm{Fc} \gamma$ receptors mediate inhibitory signal by recruiting phosphatases. ${ }^{21,22)}$

In this study, we found that Syk was active mainly in $B$ cells in human AAA tissue, suggesting that infiltrating B cells are immunologically active, although exactly how B cells and Syk are activated and their function remain to be investigated. It should be noted that the suppression of MMP-9 and IL-6 secretions by the Syk inhibitor was not detected at the baseline but was observed when AAA tis- 
sue was stimulated with IgG. Because AAA tissue secreted detectable amount of MMP-9 and IL-6 in the tissue culture condition, this unstimulated secretion seemed independent of Syk activity even though histological analysis showed Syk activation in inflammatory cells. On the other hand, IgG-stimulated secretions of MMP-9 and IL-6 were sensitive to the Syk inhibitor, suggesting that exogenous IgG stimulated Syk in AAA tissue. This could be due to the fact that Syk activation may be diminished during several days of tissue culture in the absence of serum or immunoglobulins. In our previous report, we showed that Syk was activated by exogenous $\operatorname{IgG}$ as demonstrated in $\mathrm{B}$ cell- and immunoglobulin-deficient $\mu \mathrm{MT}$ mice, ${ }^{7)}$ suggesting that activation of Syk and secretions of IL- 6 and MMP-9 were dynamically regulated by IgG in AAA tissue. Although we intended to detect the Syk activation in the cultured AAA tissue by western blotting and immunostaining, our attempt was unsuccessful possibly due to the tissue degradation activity in the culture condition. Also, whether any risk factors for AAA such as smoking or other clinical conditions would alter the response to IgG or the Syk activity in AAA tissue remains to be clarified in future research. Nonetheless, our data indicate that Syk inhibition is effective in suppressing tissue inflammatory and destructive activities in the presence of IgG.

\section{Therapeutic implication}

Considering the clinical situations, several challenges must be overcome in pharmacological therapy for AAA.,3) Because most of AAA patients do not have symptoms for several years, the therapy may need to be continued for long time with minimal side effect. Ideally, the pharmacological therapy may be applied to the patients with high activity of tissue inflammation and destruction in AAA tissue but discontinued for patients with low disease activity. This strategy would not only minimize the potential side effect but also reduce the medical cost of the pharmacotherapy. As symptom cannot be an indicator for the disease activity in AAA, sensitive and reliable biomarkers need to be identified to realize the pharmacological therapy for AAA. Whether inhibition of Syk suffices these requirements remains to be clarified.

\section{Conclusion}

In conclusion, our data suggested that tissue inflammatory and destructive activities may be regulated by IgG, which depends on Syk activity. Future studies should be directed on whether endogenous IgG participates in AAA pathogenesis in a Syk-dependent manner, how Syk is activated in human AAA, and whether and how Syk inhibition therapy can be realized in the clinical practice.

\section{Acknowledgments}

This work was funded in part by grants JSPS KAKENHI $18 \mathrm{~K} 16406$ (to RK), 17K10770 (to AF), and 16H05428 (to HA). We would like to thank Ms. Kiyohiro, Ms. Nishigata, Ms. Nakao, Ms. Shiramizu, Ms. Nakayama, and Dr. Yamamoto for technical assistance.

\section{Disclosure Statements}

The authors declare no conflict of financial interest concerning this manuscript.

\section{Author Contributions}

Study conception: RK, HA, AF, YF, HT

Data collection: RK, HA, AF, HO, YS, ST, SH, HT

Analysis: RK, HA, AF

Writing: RK, HA

Funding acquisition: RK, HA, AF

Critical review and revision: all authors

Final approval of the article: all authors

Accountability for all aspects of the work: all authors

\section{References}

1) Cronenwett JL, Johnston KW. Vascular Surgery. 8th ed. Philadelphia: Elsevier Health Sciences, 2014.

2) Hirsch AT, Haskal ZJ, Hertzer NR, et al. ACC/AHA 2005 Practice guidelines for the management of patients with peripheral arterial disease (lower extremity, renal, mesenteric, and abdominal aortic). Circulation 2006; 113: e463-654.

3) Aoki H, Yoshimura K, Matsuzaki M. Turning back the clock: regression of abdominal aortic aneurysms via pharmacotherapy. J Mol Med (Berl) 2007; 85: 1077-88.

4) Emeto TI, Seto SW, Golledge J. Targets for medical therapy to limit abdominal aortic aneurysm progression. Curr Drug Targets 2014; 15: 860-73.

5) Zhou HF, Yan H, Bertram P, et al. Fibrinogen-specific antibody induces abdominal aortic aneurysm in mice through complement lectin pathway activation. Proc Natl Acad Sci USA 2013; 110: E4335-44.

6) Zhou HF, Yan H, Stover CM, et al. Antibody directs properdin-dependent activation of the complement alternative pathway in a mouse model of abdominal aortic aneurysm. Proc Natl Acad Sci USA 2012; 109: E415-22.

7) Furusho A, Aoki H, Ohno-Urabe S, et al. Involvement of $\mathrm{B}$ cells, immunoglobulins, and Syk in the pathogenesis of abdominal aortic aneurysm. J Am Heart Assoc 2018; 7: $\mathrm{e} 007750$.

8) Ohno T, Aoki H, Ohno S, et al. Cytokine profile of human abdominal aortic aneurysm: involvement of JAK/STAT pathway. Ann Vasc Dis 2018; 11: 84-90.

9) Anderson DR, Grillo-Lopez A, Varns C, et al. Targeted anticancer therapy using rituximab, a chimaeric anti-CD20 antibody (IDEC-C2B8) in the treatment of non-Hodgkin's B-cell lymphoma. Biochem Soc Trans 1997; 25: 705-8. 
10) Deng GM, Kyttaris VC, Tsokos GC. Targeting Syk in autoimmune rheumatic diseases. Front Immunol 2016; 7: 78.

11) Kurosaki T, Hikida M. Tyrosine kinases and their substrates in B lymphocytes. Immunol Rev 2009; 228: 132-48.

12) Daeron M. Fc receptor biology. Annu Rev Immunol 1997; 15: 203-34.

13) Geahlen RL. Getting Syk: spleen tyrosine kinase as a therapeutic target. Trends Pharmacol Sci 2014; 35: 414-22.

14) Senemaud J, Caligiuri G, Etienne H, et al. Translational relevance and recent advances of animal models of abdominal aortic aneurysm. Arterioscler Thromb Vasc Biol 2017; 37 : 401-10.

15) Yoshimura K, Aoki H, Ikeda Y, et al. Regression of abdominal aortic aneurysm by inhibition of c-Jun N-terminal kinase. Nat Med 2005; 11: 1330-8.

16) Ocana E, Bohorquez JC, Perez-Requena J, et al. Characterisation of $\mathrm{T}$ and $\mathrm{B}$ lymphocytes infiltrating abdominal aortic aneurysms. Atherosclerosis 2003; 170: 39-48.
17) Zhang L, Wang Y. B lymphocytes in abdominal aortic aneurysms. Atherosclerosis 2015; 242: 311-7.

18) Shimizu K, Mitchell RN, Libby P. Inflammation and cellular immune responses in abdominal aortic aneurysms. Arterioscler Thromb Vasc Biol 2006; 26: 987-94.

19) Pagano MB, Zhou HF, Ennis TL, et al. Complementdependent neutrophil recruitment is critical for the development of elastase-induced abdominal aortic aneurysm. Circulation 2009; 119: 1805-13.

20) Cornall RJ, Cheng AM, Pawson T, et al. Role of Syk in B-cell development and antigen-receptor signaling. Proc Natl Acad Sci USA 2000; 97: 1713-8.

21) Takai T. Roles of Fc receptors in autoimmunity. Nat Rev Immunol 2002; 2: 580-92.

22) Yi YS, Son YJ, Ryou C, et al. Functional roles of Syk in macrophage-mediated inflammatory responses. Mediators Inflamm 2014; 2014: 270302. 